\title{
IMPLICIT DIFFERENCE METHODS FOR QUASILINEAR PARABOLIC FUNCTIONAL DIFFERENTIAL PROBLEMS OF THE DIRICHLET TYPE
}

Abstract. Classical solutions of quasilinear functional differential equations are approximated with solutions of implicit difference schemes. Proofs of convergence of the difference methods are based on a comparison technique. Nonlinear estimates of the Perron type with respect to the functional variable for given functions are used. Numerical examples are given.

1. Introduction. For any two metric spaces $X$ and $Y$ we denote by $C(X, Y)$ the class of all continuous functions defined on $X$ and taking values in $Y$. Let $M_{n \times n}$ denote the set of all $n \times n$ real matrices. We will use vectorial inequalities, understanding that the same inequalities hold between the corresponding components. Let

$$
E=[0, a] \times(-b, b), \quad D=\left[-d_{0}, 0\right] \times[-d, d],
$$

where $a>0, b=\left(b_{1}, \ldots, b_{n}\right), b_{i}>0$ for $1 \leq i \leq n, d_{0} \in \mathbb{R}_{+}, d=$ $\left(d_{1}, \ldots, d_{n}\right) \in \mathbb{R}_{+}^{n}$ and $\mathbb{R}_{+}=[0, \infty)$. We put $c=\left(c_{1}, \ldots, c_{n}\right)=b+d$ and $\partial_{0} E=[0, a] \times([-c, c] \backslash(-b, b)), \quad E_{0}=\left[-d_{0}, 0\right] \times[-c, c], \quad \Omega=E \cup E_{0} \cup \partial_{0} E$. For a function $z: \Omega \rightarrow \mathbb{R}$ and a point $(t, x) \in[0, a] \times[-b, b]$ we define a function $z_{(t, x)}: D \rightarrow \mathbb{R}$ as follows:

$$
z_{(t, x)}(\xi, y)=z(t+\xi, x+y) \quad \text { for }(\xi, y) \in D .
$$

The function $z_{(t, x)}$ is the restriction of $z$ to the set $\left[t-d_{0}, t\right] \times[x-d, x+d]$ and this restriction is shifted to the set $D$. Elements of the space $C(D, \mathbb{R})$ will be denoted by $w, \bar{w}$ and so on. Write $\Sigma=E \times C(D, \mathbb{R})$ and suppose

2000 Mathematics Subject Classification: 65M12, 35R10.

Key words and phrases: functional differential equations, implicit difference methods, stability and convergence. 
that the functions

$$
\begin{gathered}
f: \Sigma \rightarrow M_{n \times n}, \quad f=\left[f_{i j}\right]_{i, j=1, \ldots, n}, \\
g: \Sigma \rightarrow \mathbb{R}^{n}, \quad g=\left(g_{1}, \ldots, g_{n}\right), \quad G: \Sigma \rightarrow \mathbb{R}
\end{gathered}
$$

and

$$
\varphi: \partial_{0} E \cup E_{0} \rightarrow \mathbb{R}, \quad \alpha: E \rightarrow \mathbb{R}^{1+n}, \quad \alpha=\left(\alpha_{0}, \alpha^{\prime}\right), \alpha^{\prime}=\left(\alpha_{1}, \ldots, \alpha_{n}\right),
$$

are given. We assume that $\alpha(t, x) \in \bar{E}$ and $\alpha_{0}(t, x) \leq t$ for $(t, x) \in E$, where $\bar{E}$ is the closure of $E$. We consider the problem consisting of the quasilinear differential functional equation

$$
\begin{aligned}
\partial_{t} z(t, x)= & \sum_{i, j=1}^{n} f_{i j}\left(t, x, z_{\alpha(t, x)}\right) \partial_{x_{i} x_{j}} z(t, x) \\
& +\sum_{i=1}^{n} g_{i}\left(t, x, z_{\alpha(t, x)}\right) \partial_{x_{i}} z(t, x)+G\left(t, x, z_{\alpha(t, x)}\right)
\end{aligned}
$$

with the initial boundary condition of the Dirichlet type

$$
z(t, x)=\varphi(t, x) \quad \text { for }(t, x) \in E_{0} \cup \partial_{0} E .
$$

We are interested in establishing a method of numerical approximation of classical solutions to (1), (2) by means of solutions of associated implicit difference schemes and in estimating the difference between the exact and approximate solutions.

Explicit difference methods for (1), (2) consist in replacing the partial derivatives $\partial_{t}, \partial_{x}=\left(\partial_{x_{1}}, \ldots, \partial_{x_{n}}\right)$ and $\partial_{x x}=\left[\partial_{x_{i} x_{j}}\right]_{i, j=1, \ldots, n}$ with difference expressions. Moreover, because equation (1) contains the functional variable $z_{\alpha(t, x)}$ which is an element of the space $C(D, \mathbb{R})$ we need interpolating operators. This leads to a difference functional equation of the Volterra type. Solutions of these equations approximate, under suitable assumptions on given functions and on the mesh, solutions of the original problem. Methods of difference inequalities or theorems on recurrent inequalities are used in the investigation of the stability of difference schemes generated by parabolic functional differential problems. The proofs of the convergence are also based on a general theorem on an error estimate for approximate solutions of functional difference equations of the Volterra type with the unknown function of several variables.

Difference methods for nonlinear parabolic differential or functional differential equations were considered by many authors under various assumptions. It is not our aim to give a full review of papers concerning convergence results for difference schemes. We only mention those which contain such reviews: [8], [11], [12], [14], [15].

Two types of assumptions are needed in theorems on convergence of explicit difference methods for (1), (2). Conditions of the first type deal 
with the regularity of the given functions and the parabolicity of (1). It is assumed that $f, g, G$ are continuous on $\Sigma$ and satisfy nonlinear estimates of the Perron type with respect to the functional variable. The parabolicity of (1) means that the matrix $f$ is symmetric on $\Sigma$ and

$$
f_{i i}(P)>\sum_{\substack{j=1 \\ j \neq i}}^{n} f_{i j}(P), \quad i=1, \ldots, n,
$$

where $P=(t, x, w) \in \Sigma$. Note that the above condition implies that

$$
\sum_{i, j=1}^{n} f_{i j}(P) \xi_{i} \xi_{j} \geq 0 \quad \text { for } \xi=\left(\xi_{1}, \ldots, \xi_{n}\right) \in \mathbb{R}^{n},
$$

which means that equation (1) is parabolic in the sense of Walter [16]. The conditions of the second type are connected with the mesh. It is assumed that

$$
1-2 h_{0} \sum_{i=1}^{n} \frac{1}{h_{i}^{2}} f_{i i}(P)+h_{0} \sum_{\substack{j=1 \\ j \neq i}}^{n} \frac{1}{h_{i} h_{j}}\left|f_{i j}(P)\right| \geq 0
$$

for $P \in \Sigma$, where $h_{0}$ and $h^{\prime}=\left(h_{1}, \ldots, h_{n}\right)$ are steps of the mesh. This is a very restrictive assumption on the relations between $h_{0}$ and $h^{\prime}$. The differential functional equation

$$
\partial_{t} z(t, x)=\sum_{i=1}^{n} \partial_{x_{i} x_{i}} z(t, x)+\sum_{i=1}^{n} \partial_{x_{i}} z(t, x) g_{i}\left(t, x, z_{\alpha(t, x)}\right)+G\left(t, x, z_{\alpha(t, x)}\right)
$$

is a particular case of (1). Condition (3) for equation (4) has the form

$$
1-2 h_{0} \sum_{i=1}^{n} \frac{1}{h_{i}^{2}} \geq 0 \text {. }
$$

The aim of the paper is to show that the restriction (3) may be omitted in the case of implicit difference schemes (9), (10).

Parabolic functional differential equations find applications in different fields of knowledge. We give a few examples. The most important classes of such problems are the Lotka-Volterra type reaction diffusion equations which include delays and integral terms [4], [5]. Some systems of delayed reaction diffusion equations have also been used in modelling genetic repression [9]. A nuclear reactor model has been described in [13] by means of a system of two parabolic equations with delays. Reaction diffusion equations with delays arise naturally in the study of climate models [6]. A mathematical description of the overall control systems may be given by a parabolic equation with a deviated time variable [17]. For an extensive bibliography 
on applications of parabolic functional differential equations see the monograph [18].

We give an example of equation which can be obtained from (1) by specializing given functions.

Example 1. Assume that $d_{0}=0, d=0$ where $\mathbf{0}=(0, \ldots, 0) \in \mathbb{R}^{n}$ and

$$
\tilde{f}: E \times \mathbb{R} \rightarrow M_{n \times n}, \quad \widetilde{f}=\left[\widetilde{f}_{i j}\right]_{i, j=1, \ldots, n},
$$

$$
\widetilde{g}: E \times \mathbb{R} \rightarrow \mathbb{R}^{n}, \quad \widetilde{g}=\left(\widetilde{g}_{1}, \ldots, \widetilde{g}_{n}\right), \quad \widetilde{G}: E \times \mathbb{R} \rightarrow \mathbb{R}
$$

are given functions. We define $f, g, G$ as follows:

$$
\begin{gathered}
f(t, x, w)=\widetilde{f}(t, x, w(0, \mathbf{0})), \\
g(t, x, w)=\widetilde{g}(t, x, w(0, \mathbf{0})), \quad G(t, x, w)=\widetilde{G}(t, x, w(0, \mathbf{0})) .
\end{gathered}
$$

Then (1) reduces to the differential equation with deviated variables

$$
\begin{aligned}
\partial_{t} z(t, x)= & \sum_{i, j=1}^{n} \widetilde{f}_{i j}(t, x, z(\alpha(t, x))) \partial_{x_{i} x_{j}} z(t, x) \\
& +\sum_{i=1}^{n} \widetilde{g}_{i}(t, x, z(\alpha(t, x))) \partial_{x_{i}} z(t, x)+\widetilde{G}(t, x, z(\alpha(t, x))) .
\end{aligned}
$$

ExAmple 2. Suppose that $\beta, \gamma: E \rightarrow \mathbb{R}^{1+n}$ and $\beta=\left(\beta_{0}, \beta^{\prime}\right), \gamma=$ $\left(\gamma_{0}, \gamma^{\prime}\right), \beta^{\prime}=\left(\beta_{1}, \ldots, \beta_{n}\right), \gamma^{\prime}=\left(\gamma_{1}, \ldots, \gamma_{n}\right)$. We assume that

$$
\begin{aligned}
-d_{0} & \leq\left(\beta_{0}-\alpha_{0}\right)(t, x) \leq 0, \quad-d_{0} \leq\left(\gamma_{0}-\alpha_{0}\right)(t, x) \leq 0, \\
-d & \leq\left(\beta^{\prime}-\alpha^{\prime}\right)(t, x) \leq d, \quad-d \leq\left(\gamma^{\prime}-\alpha^{\prime}\right)(t, x) \leq d,
\end{aligned}
$$

where $(t, x) \in E$. Write

$$
I[w](t, x)=\int_{(\beta-\alpha)(t, x)}^{(\gamma-\alpha)(t, x)} w(\tau, y) d y d \tau .
$$

For the above $\widetilde{f}, \widetilde{g}, \widetilde{G}$ we put

$$
\begin{aligned}
& f(t, x, w)=\widetilde{f}(t, x, I[w](t, x)), \\
& g(t, x, w)=\widetilde{g}(t, x, I[w](t, x)), \quad G(t, x, w)=\widetilde{G}(t, x, I[w](t, x)) .
\end{aligned}
$$

Then (1) is equivalent to the differential integral equation

$$
\begin{gathered}
\partial_{t} z(t, x)=\sum_{i, j=1}^{n} \widetilde{f}_{i j}\left(t, x, \int_{\beta(t, x)}^{\gamma(t, x)} z(\tau, y) d y d \tau\right) \partial_{x_{i} x_{j}} z(t, x) \\
+\sum_{i=1}^{n} \widetilde{g}_{i}\left(t, x, \int_{\beta(t, x)}^{\gamma(t, x)} z(\tau, y) d y d \tau\right) \partial_{x_{i}} z(t, x)+\widetilde{G}\left(t, x, \int_{(\beta)(t, x)}^{(\gamma)(t, x)} z(\tau, y) d y d \tau\right) .
\end{gathered}
$$


It is clear that more complicated differential equations with deviated variables and differential integral equations can be derived from (1). Note also that equations (5), (6) cannot be obtained as particular cases of differential functional equations considered in [2], [3], [8]. The right hand sides of equations in those papers depend on the restrictions of $z$ to the set $\left[t-d_{0}, t\right] \times[x-d, x+d]$. In our paper, the differential functional equations depend on the restriction of $z$ to $\left[\alpha_{0}(t, x)-d_{0}, \alpha_{0}(t, x)\right] \times\left[\alpha^{\prime}(t, x)-d, \alpha^{\prime}(t, x)+d\right]$.

The paper is organized as follows. In Section 2 we construct a class of implicit difference schemes for (1), (2). The existence and uniqueness of approximate solutions, which is not obvious in contrast to explicit methods, are proved in Section 3. In Section 4, which is the main part of the paper, we give sufficient conditions for convergence of the implicit difference schemes. Finally, numerical examples are presented in the last part of the paper.

2. Discretization of mixed problems. We formulate a difference problem corresponding to (1), (2). We denote by $\mathbb{N}$ and $\mathbb{Z}$ the set of natural numbers and the set of integers, respectively. Let $F(X, Y)$ denote the class of all functions defined on $X$ and taking values in $Y$ where $X$ and $Y$ are arbitrary sets. For $x \in \mathbb{R}^{n}, U \in M_{n \times n}$, where $x=\left(x_{1}, \ldots, x_{n}\right), U=\left[u_{i j}\right]_{i, j=1, \ldots, n}$, we write

$$
\|x\|=\sum_{i=1}^{n}\left|x_{i}\right|, \quad\|U\|_{*}=\sum_{i, j=1}^{n}\left|u_{i j}\right| .
$$

We define a mesh on $\Omega$ in the following way. Let $\left(h_{0}, h^{\prime}\right)$ where $h^{\prime}=$ $\left(h_{1}, \ldots, h_{n}\right)$ stand for steps of the mesh. For $h=\left(h_{0}, h^{\prime}\right)$ and $(r, m) \in \mathbb{Z}^{1+n}$ where $m=\left(m_{1}, \ldots, m_{n}\right)$ we define nodal points as follows:

$$
t^{(r)}=r h_{0}, \quad x^{(m)}=\left(x_{1}^{\left(m_{1}\right)}, \ldots, x_{n}^{\left(m_{n}\right)}\right)=\left(m_{1} h_{1}, \ldots, m_{n} h_{n}\right) .
$$

Denote by $H$ the set of all $h=\left(h_{0}, h^{\prime}\right)$ such that there exist $K_{0} \in \mathbb{Z}$ and $K=\left(K_{1}, \ldots, K_{n}\right) \in \mathbb{Z}^{n}$ satisfying the conditions $K_{0} h_{0}=d_{0}$ and $\left(K_{1} h_{1}, \ldots, K_{n} h_{n}\right)=c$. Let $N \in \mathbb{N}$ be defined by the relations $N h_{0} \leq a<$ $(N+1) h_{0}$. For $h \in H$ we put

$$
\mathbb{R}_{h}^{1+n}=\left\{\left(t^{(r)}, x^{(m)}\right):(r, m) \in \mathbb{Z}^{1+n}\right\}
$$

and

$$
\begin{aligned}
E_{h} & =E \cap \mathbb{R}_{h}^{1+n}, \quad E_{h}^{\prime}=\left\{\left(t^{(r)}, x^{(m)}\right): 0 \leq r \leq N-1,-K \leq m \leq K\right\}, \\
E_{0 . h} & =E_{0} \cap \mathbb{R}_{h}^{1+n}, \quad \partial_{0} E_{h}=\partial_{0} E \cap \mathbb{R}_{h}^{1+n}, \\
\Omega_{h} & =\Omega \cap \mathbb{R}_{h}^{1+n}=E_{h} \cup E_{0 . h} \cup \partial_{0} E_{h} .
\end{aligned}
$$

System (1) contains the functional variable $z_{\alpha(t, x)}$ which is an element of the space $C(D, \mathbb{R})$. Thus we need an interpolating operator $T_{h}: F\left(\Omega_{h}, \mathbb{R}\right) \rightarrow$ 
$C(\Omega, \mathbb{R})$. We define $T_{h}$ in the following way. Suppose that $z \in F\left(\Omega_{h}, \mathbb{R}\right)$ and $(t, x) \in \Omega$. We consider two possibilities.

A) Suppose that there exists a point $\left(t^{(r)}, x^{(m)}\right) \in \Omega_{h}$ such that $\left(t^{(r+1)}\right.$, $\left.x^{(m+1)}\right) \in \Omega_{h}$, where $m+1=\left(m_{1}+1, \ldots, m_{n}+1\right)$ and $t^{(r)} \leq t \leq t^{(r+1)}$, $x^{(m)} \leq x \leq x^{(m+1)}$. Put

$$
\Im=\left\{\lambda=\left(\lambda_{1}, \ldots, \lambda_{n}\right): \lambda_{i} \in\{0,1\} \text { for } 0 \leq i \leq n\right\} .
$$

We define

$$
\begin{aligned}
T_{h}[z](t, x)= & \frac{t-t^{(r)}}{h_{0}} \sum_{\lambda \in \Im} z^{(r+1, m+\lambda)}\left(\frac{x-x^{(m)}}{h^{\prime}}\right)^{\lambda}\left(1-\frac{x-x^{(m)}}{h^{\prime}}\right)^{1-\lambda} \\
& +\left(1-\frac{t-t^{(r)}}{h_{0}}\right) \sum_{\lambda \in \Im} z^{(r, m+\lambda)}\left(\frac{x-x^{(m)}}{h^{\prime}}\right)^{\lambda}\left(1-\frac{x-x^{(m)}}{h^{\prime}}\right)^{1-\lambda}
\end{aligned}
$$

where

$$
\begin{gathered}
\left(\frac{x-x^{(m)}}{h^{\prime}}\right)^{\lambda}=\prod_{i=1}^{n}\left(\frac{x_{i}-x_{i}^{\left(m_{i}\right)}}{h_{i}}\right)^{\lambda_{i}}, \\
\left(1-\frac{x-x^{(m)}}{h^{\prime}}\right)^{1-\lambda}=\prod_{i=1}^{n}\left(1-\frac{x_{i}-x_{i}^{\left(m_{i}\right)}}{h_{i}}\right)^{1-\lambda_{i}}
\end{gathered}
$$

and we take $0^{0}=1$ in the above formulas.

B) If $(t, x) \in \Omega$ and $t^{(N)}<t \leq a$, we define $T_{h}[z](t, x)=T_{h}[z]\left(t^{(N)}, x\right)$.

Thus we have defined $T_{h}[z]$ on $\Omega$ and $T_{h}[z] \in C(\Omega, \mathbb{R})$.

The above interpolating operator was first proposed in [7] for the construction of explicit difference schemes related to first order partial differential functional equations.

Let $z: \Omega_{h} \rightarrow \mathbb{R}$ and $\left(t^{(r)}, x^{(m)}\right) \in E_{h}^{\prime}$, and define

$$
\begin{aligned}
& \delta_{i}^{+} z^{(r, m)}=\frac{1}{h_{i}}\left(z^{\left(r, m+e_{i}\right)}-z^{(r, m)}\right), \\
& \delta_{i}^{-} z^{(r, m)}=\frac{1}{h_{i}}\left(z^{(r, m)}-z^{\left(r, m-e_{i}\right)}\right), \quad 1 \leq i \leq n,
\end{aligned}
$$

and

$$
\begin{aligned}
\delta_{0} z^{(r, m)} & =\frac{1}{h_{0}}\left(z^{(r+1, m)}-z^{(r, m)}\right), \\
\delta_{i} z^{(r+1, m)} & =\frac{1}{2}\left(\delta_{i}^{+} z^{(r+1, m)}+\delta_{i}^{-} z^{(r+1, m)}\right), \quad 1 \leq i \leq n .
\end{aligned}
$$

Put $J=\left\{(i, j) \in \mathbb{N}^{2}: 1 \leq i, j \leq n, i \neq j\right\}$. Suppose that a function $\varphi_{h}: E_{0 . h} \cup \partial_{0} E_{h} \rightarrow \mathbb{R}$ is given. We approximate solutions of (1)-(2) with 
solutions of the difference functional equation

$$
\begin{aligned}
\delta_{0} z^{(r, m)}= & \sum_{i, j=1}^{n} f_{i j}\left(P^{(r, m)}[z]\right) \delta_{i j} z^{(r+1, m)} \\
& +\sum_{i=1}^{n} g_{i}\left(P^{(r, m)}[z]\right) \delta_{i} z^{(r+1, m)}+G\left(P^{(r, m)}[z]\right),
\end{aligned}
$$

with the initial boundary condition

$$
z^{(r, m)}=\varphi_{h}^{(r, m)} \quad \text { on } E_{0 . h} \cup \partial_{0} E_{h},
$$

where $P^{(r, m)}[z]=\left(t^{(r)}, x^{(m)},\left(T_{h}[z]\right)_{\alpha^{(r, m)}}\right), \alpha^{(r, m)}=\alpha\left(t^{(r)}, x^{(m)}\right)$. The difference operator $\delta^{(2)}=\left[\delta_{i j}\right]_{i, j=1, \ldots, n}$ is defined in the following way:

$$
\begin{array}{ll}
\delta_{i i} z^{(r+1, m)}=\delta_{i}^{+} \delta_{i}^{-} z^{(r+1, m)}, \quad 1 \leq i \leq n, & \\
\delta_{i j} z^{(r+1, m)}=\frac{1}{2}\left(\delta_{i}^{+} \delta_{j}^{-} z^{(r+1, m)}+\delta_{i}^{-} \delta_{j}^{+} z^{(r+1, m)}\right) & \text { if } f_{i j}\left(P^{(r, m)}[z]\right) \leq 0, \\
\delta_{i j} z^{(r+1, m)}=\frac{1}{2}\left(\delta_{i}^{+} \delta_{j}^{+} z^{(r+1, m)}+\delta_{i}^{-} \delta_{j}^{-} z^{(r+1, m)}\right) & \text { if } f_{i j}\left(P^{(r, m)}[z]\right)>0 .
\end{array}
$$

The difference functional problem (9)-(10) with $\delta_{0}, \delta, \delta^{(2)}$ defined by (7), (8), (11)-(13) is viewed as an implicit difference method for (1)-(2). It is important in our considerations that the difference expressions $\delta z$ and $\delta^{(2)} z$ appear in (9) at the point $\left(t^{(r+1)}, x^{(m)}\right)$. It follows from $(12),(13)$ that the definition of the difference expressions $\delta_{i j} z^{(r+1, m)}$ for $(i, j) \in J$ depends on the sign of $f_{i j}\left(P^{(r, m)}[z]\right)$. The corresponding explicit difference scheme consists of the difference functional equation

$$
\begin{aligned}
\delta_{0} z^{(r, m)}= & \sum_{i, j=1}^{n} f_{i j}\left(P^{(r, m)}[z]\right) \delta_{i j} z^{(r, m)} \\
& +\sum_{i=1}^{n} g_{i}\left(P^{(r, m)}[z]\right) \delta_{i} z^{(r, m)}+G\left(P^{(r, m)}[z]\right),
\end{aligned}
$$

with the initial boundary condition (10). It is clear that there exists exactly one solution of problem (10), (14). Sufficient conditions for the convergence of the difference scheme (10), (14) can be deduced from [2].

We prove that under natural assumptions on given functions there exists exactly one solution $u_{h}: E_{h} \rightarrow \mathbb{R}$ of the implicit difference problem (9), (10). In Section 4 we prove a convergence result.

3. Solutions of difference functional problems. For a function $z$ : $E_{h} \rightarrow \mathbb{R}$ and a point $\left(t^{(r)}, x^{(m)}\right) \in E_{h}$ we put

$$
J_{-}^{(r, m)}[z]=\left\{(i, j) \in J: f_{i j}\left(P^{(r, m)}[z]\right) \leq 0\right\}, \quad J_{+}^{(r, m)}[z]=J \backslash J_{-}^{(r, m)}[z] .
$$


Assumption $\mathrm{H}[f, g, \alpha]$.

1) The functions $f: \Sigma \rightarrow M[n]$ and $g: \Sigma \rightarrow \mathbb{R}^{n}$ are continuous on $\Sigma$ and

$$
-\frac{1}{2}\left|g_{i}(P)\right|+\frac{1}{h_{i}} f_{i i}(P)-\sum_{\substack{j=1 \\ j \neq i}}^{n} \frac{1}{h_{j}}\left|f_{i j}(P)\right| \geq 0, \quad i=1, \ldots, n,
$$

for $P=(t, x, w) \in \Sigma$.

2) $\alpha \in C\left(E, \mathbb{R}^{1+n}\right), \alpha=\left(\alpha_{0}, \alpha^{\prime}\right), \alpha(t, x) \in E$ and $\alpha_{0}(t, x) \leq t$ for $(t, x) \in E$.

REMARK 1. Suppose that

$$
f_{i i}(P)-\sum_{\substack{j=1 \\ j \neq i}}^{n}\left|f_{i j}(P)\right| \geq \varepsilon, \quad P \in \Sigma,
$$

where $\varepsilon>0$, and $h_{1}=\cdots=h_{n}$ are sufficiently small. Then condition (16) is satisfied.

ThEOREM 3.1. If Assumption $\mathrm{H}[f, g, \alpha]$ is satisfied and $\varphi_{h}: \partial_{0} E_{h} \cup$ $E_{0 . h} \rightarrow \mathbb{R}$, then there is exactly one solution $u_{h}: E_{h} \rightarrow \mathbb{R}$ of problem (9)-(10).

Proof. Suppose that $0 \leq r \leq N-1$ is fixed and that the solution $u_{h}$ of (9), (10) is defined on $\Omega_{h} \cap\left(\left[-d_{0}, t^{(r)}\right] \times \mathbb{R}^{n}\right)$. We prove that the numbers $u_{h}^{(r+1, m)}$, where $\left(t^{(r+1)}, x^{(m)}\right) \in E_{h}$, exist and are unique. There is $Q_{h}>0$ such that

$$
Q_{h} \geq 2 h_{0} \sum_{i=1}^{n} \frac{1}{h_{i}^{2}} f_{i i}\left(P^{(r, m)}\left[u_{h}\right]\right)-h_{0} \sum_{(i, j) \in J} \frac{1}{h_{i} h_{j}}\left|f_{i j}\left(P^{(r, m)}\left[u_{h}\right]\right)\right| .
$$

Problem (9)-(10) is equivalent to the system of equations

$$
\begin{aligned}
z^{(r+1, m)}= & \frac{1}{Q_{h}+1}\left[Q_{h} z_{h}^{(r+1, m)}+u_{h}^{(r, m)}+h_{0} \sum_{i, j=1}^{n} f_{i j}\left(P^{(r, m)}\left[u_{h}\right]\right) \delta_{i j} z^{(r+1, m)}\right. \\
& \left.+h_{0} \sum_{i=1}^{n} g_{i}\left(P^{(r, m)}\left[u_{h}\right]\right) \delta_{i} z^{(r+1, m)}+h_{0} G\left(P^{(r, m)}\left[u_{h}\right]\right)\right]
\end{aligned}
$$

with the boundary condition

$$
z^{(r+1, m)}=\varphi_{h}^{(r+1, m)} \quad \text { for }\left(t^{(r+1)}, x^{(m)}\right) \in \partial_{0} E_{h},
$$


where $z^{(r+1, m)},-K \leq m \leq K$, are unknown. Write

$$
S_{h}=\left\{x^{(m)}:-K \leq m \leq K\right\}, \quad \partial_{0} S_{h}=\left\{x^{(m)}:\left(t^{(r+1)}, x^{(m)}\right) \in \partial_{0} E_{h}\right\} .
$$

We consider the space $F\left(S_{h}, \mathbb{R}\right)$. Elements of $F\left(S_{h}, \mathbb{R}\right)$ are denoted by $\xi, \bar{\xi}$. For $\xi \in F\left(S_{h}, \mathbb{R}\right)$ we write $\xi^{(m)}=\xi\left(x^{(m)}\right)$ and

$$
\delta \xi^{(m)}=\left(\delta_{1} \xi^{(m)}, \ldots, \delta_{n} \xi^{(m)}\right), \quad \delta^{(2)} \xi^{(m)}=\left[\delta_{i j} \xi^{(m)}\right]_{i, j=1, \ldots, n},
$$

where $\delta_{i}$ and $\delta_{i j}, 1 \leq i, j \leq n$, are defined by (8)-(13). The norm in $F\left(S_{h}, \mathbb{R}\right)$ is defined by

$$
\|\xi\|_{\infty}=\max \left\{\left|\xi^{(m)}\right|: x^{(m)} \in S_{h}\right\} .
$$

We consider the linear operator $U_{h}: F\left(S_{h}, \mathbb{R}\right) \rightarrow F\left(S_{h}, \mathbb{R}\right)$ defined by

$$
\begin{aligned}
U_{h}[\xi]^{(m)}= & \frac{1}{Q_{h}+1}\left[Q_{h} \xi^{(m)}+h_{0} \sum_{i, j=1}^{n} f_{i j}\left(P^{(r, m)}\left[u_{h}\right]\right) \delta_{i j} \xi^{(m)}\right. \\
& \left.+h_{0} \sum_{i=1}^{n} g_{i}\left(P^{(r, m)}\left[u_{h}\right]\right) \delta_{i} \xi^{(m)}\right] \quad \text { for } x^{(m)} \in S_{h} \backslash \partial_{0} S_{h},
\end{aligned}
$$

and

$$
U_{h}[\xi]^{(m)}=0 \quad \text { for } x^{(m)} \in \partial_{0} E_{h} .
$$

We prove that for $\xi \in F\left(S_{h}, \mathbb{R}\right)$ we have

$$
\left\|U_{h}[\xi]\right\|_{\infty} \leq \frac{Q_{h}}{1+Q_{h}}\|\xi\|_{\infty}
$$

Write

$$
\begin{aligned}
A_{i .+}^{(r, m)}[z]= & \frac{h_{0}}{2 h_{i}} g_{i}\left(P^{(r, m)}[z]\right)+\frac{h_{0}}{h_{i}^{2}} f_{i i}\left(P^{(r, m)}[z]\right) \\
& -\sum_{\substack{j=1 \\
j \neq i}}^{n} \frac{h_{0}}{h_{i} h_{j}}\left|f_{i j}\left(P^{(r, m)}[z]\right)\right|, \\
A_{i .-}^{(r, m)}[z]= & -\frac{h_{0}}{2 h_{i}} g_{i}\left(P^{(r, m)}[z]\right)+\frac{h_{0}}{h_{i}^{2}} f_{i i}\left(P^{(r, m)}[z]\right) \\
& -\sum_{\substack{j=1 \\
j \neq i}}^{n} \frac{h_{0}}{h_{i} h_{j}}\left|f_{i j}\left(P^{(r, m)}[z]\right)\right|, \\
A^{(r, m)}[z]= & -2 \sum_{i=1}^{n} \frac{h_{0}}{h_{i}^{2}} f_{i i}\left(P^{(r, m)}[z]\right)+\sum_{(i, j) \in J}^{n} \frac{h_{0}}{h_{i} h_{j}}\left|f_{i j}\left(P^{(r, m)}[z]\right)\right|,
\end{aligned}
$$


where $1 \leq i \leq n$. It follows from Assumption $\mathrm{H}[f, g, \alpha]$ that

$$
\begin{aligned}
\left.\mid U_{h}[\xi]\right]^{(m)} \mid & \left(Q_{h}+1\right) \\
\leq & \left|\left(Q_{h}+A^{(r, m)}\left[u_{h}\right]\right) \xi^{(m)}\right| \\
& +\left|\sum_{i=1}^{n} A_{i .+}^{(r, m)}\left[u_{h}\right] \xi^{\left(m+e_{i}\right)}\right|+\left|\sum_{i=1}^{n} A_{i .-}^{(r, m)}\left[u_{h}\right] \xi^{\left(m-e_{i}\right)}\right| \\
& +h_{0} \sum_{(i, j) \in J_{+}^{(r, m)}\left[u_{h}\right]}^{n} \frac{1}{2 h_{i} h_{j}} f_{i j}\left(P^{(r, m)}\left[u_{h}\right]\right)\left[\left|\xi^{\left(m+e_{i}+e_{j}\right)}\right|+\left|\xi^{\left(m-e_{i}-e_{j}\right)}\right|\right] \\
& -h_{0} \sum_{(i, j) \in J_{-}^{(r, m)}\left[u_{h}\right]}^{n} \frac{1}{2 h_{i} h_{j}} f_{i j}\left(P^{(r, m)}\left[u_{h}\right]\right)\left[\left|\xi^{\left(m+e_{i}-e_{j}\right)}\right|+\left|\xi^{\left(m-e_{i}+e_{j}\right)}\right|\right] .
\end{aligned}
$$

We conclude from Assumption $\mathrm{H}[f, g, \alpha]$ and from (18) that

$$
Q_{h}+A^{(r, m)}\left[u_{h}\right] \geq 0, \quad A_{i .+}^{(r, m)}\left[u_{h}\right] \geq 0, \quad A_{i .-}^{(r, m)}\left[u_{h}\right] \geq 0, \quad 1 \leq i \leq n,
$$

and

$$
A^{(r, m)}\left[u_{h}\right]+\sum_{i=1}^{n} A_{i .+}^{(r, m)}\left[u_{h}\right]+\sum_{i=1}^{n} A_{i .-}^{(r, m)}\left[u_{h}\right]
$$

$+h_{0} \sum_{(i, j) \in J_{+}^{(r, m)}\left[u_{h}\right]}^{n} \frac{1}{h_{i} h_{j}} f_{i j}\left(P^{(r, m)}\left[u_{h}\right]\right)-h_{0} \sum_{(i, j) \in J_{-}^{(r, m)}\left[u_{h}\right]}^{n} \frac{1}{h_{i} h_{j}} f_{i j}\left(P^{(r, m)}\left[u_{h}\right]\right)=0$.

The above considerations and (21) imply

$$
\left|U_{h}[\xi]^{(m)}\right|\left(Q_{h}+1\right) \leq Q_{h}\|\xi\|_{\infty} \quad \text { for }-K \leq m \leq K .
$$

This completes the proof of (22). It follows that the norm of the operator $U_{h}$ is less than 1 . Thus there exists exactly one solution of (19). Since $u_{h}$ is given on the initial boundary set $\partial_{0} E_{h} \cup E_{0 . h}$, the proof is completed by induction with respect to $r, 0 \leq r \leq N$.

4. Convergence of implicit difference schemes. Write $I=\left[-d_{0}, 0\right]$. We will need the operator $V: C(D, \mathbb{R}) \rightarrow C\left(I, \mathbb{R}_{+}\right)$defined by

$$
V[w](t)=\max \{|w(t, x)|: x \in[-d, d]\}, \quad t \in I .
$$

Let $\|\cdot\|_{D}$ denote the maximum norm in the space $C(D, \mathbb{R})$. Set

$$
I_{h}=\left\{t^{(r)}:-K_{0} \leq r \leq 0\right\}, \quad A_{h}=\left\{t^{(r)}: 0 \leq r \leq N\right\} .
$$

For $\xi: I_{h} \cup A_{h} \rightarrow \mathbb{R}$ we write $\xi^{(r)}=\xi\left(t^{(r)}\right)$. If $\xi: I_{h} \cup A_{h} \rightarrow \mathbb{R}$ and $t^{(r)} \in A_{h}$ then $\xi_{[r]}: I_{h} \rightarrow \mathbb{R}$ is defined by

$$
\xi_{[r]}(\tau)=\xi\left(t^{(r)}+\tau\right), \quad \tau \in I_{h}
$$


We will need the operator $T_{h_{0}}: F\left(I_{h}, \mathbb{R}\right) \rightarrow C(I, \mathbb{R})$ defined by

$$
T_{h_{0}}[\xi](t)=\xi^{(r+1)} \frac{t-t^{(r)}}{h_{0}}+\xi^{(r)}\left(1-\frac{t-t^{(r)}}{h_{0}}\right), \quad t^{(r)} \leq t \leq t^{(r+1)},
$$

where $\xi \in F\left(I_{h}, \mathbb{R}\right)$.

It is clear that $T_{h_{0}}$ is a particular case of $T_{h}$.

Lemma 1. Suppose that $\widetilde{z}: \Omega \rightarrow \mathbb{R}$ and

1) $\widetilde{z}(t, \cdot):[-c, c] \rightarrow \mathbb{R}$ is of class $C^{2}$ for $t \in\left[-d_{0}, a\right]$ and $\widetilde{z}(\cdot, x):$ $\left[-d_{0}, a\right] \rightarrow \mathbb{R}$ is of class $C^{1}$ for $x \in[-c, c]$,

2) the constants $\widetilde{d}, \widetilde{d}_{0} \in \mathbb{R}_{+}$are defined by the relations

$$
\begin{aligned}
& \left|\partial_{x_{i} x_{j}} \widetilde{z}(t, x)\right| \leq \widetilde{d} \quad \text { for }(t, x) \in \Omega, i, j=1, \ldots, n, \\
& \left|\partial_{t} \widetilde{z}(t, x)\right| \leq \widetilde{d}_{0} \quad \text { for }(t, x) \in \Omega,
\end{aligned}
$$

Then

$$
\left|T_{h}\left(\widetilde{z}_{h}\right)_{[r, m]}-\widetilde{z}^{(r, m)}\right| \leq \widetilde{d}_{0} h_{0}+\widetilde{d}\left\|h^{\prime}\right\|^{2}, \quad\left(t^{(r)}, x^{(m)}\right) \in E_{h},
$$

where $\widetilde{z}_{h}$ is the restriction of $\widetilde{z}$ to the set $\Omega_{h}$.

The above lemma follows from Theorem 5.27 in [7]. The estimate (29) states that the function is approximated by $T_{h}\left[\widetilde{z}_{h}\right]$ and the error of this approximation is bounded by $\widetilde{d}_{0} h_{0}+\widetilde{d}\left\|h^{\prime}\right\|^{2}$.

Now we formulate assumptions on the regularity of $G, f, g$ with respect to the functional variables.

Assumption $\mathrm{H}[\sigma, f, g, G]$. The functions $f, g$ satisfy Assumption $\mathrm{H}[f, g, \alpha]$ and

1) there is $\sigma:[0, a] \times C(I, \mathbb{R}) \rightarrow \mathbb{R}_{+}$such that

(i) $\sigma$ is continuous, nondecreasing with respect to both variables and $\sigma(t, \theta)=0$ for $t \in[0, a]$, where $\theta \in C\left(I, \mathbb{R}_{+}\right)$is given by $\theta(\tau)=0$ for $\tau \in I$,

(ii) for each $c \geq 1$ the function $\widetilde{\omega}(t)=0$ for $t \in I \cup[0, a]$ is the maximal solution of the Cauchy problem

$$
\zeta^{\prime}(t)=c \sigma\left(t, \zeta_{t}\right), \quad \zeta(t)=0 \quad \text { for } t \in I,
$$

2) the estimates

$$
\begin{aligned}
\|f(t, x, w)-f(t, x, \bar{w})\|_{*} & \leq \sigma(t, V[w-\bar{w}]), \\
\|g(t, x, w)-g(t, x, \bar{w})\| & \leq \sigma(t, V[w-\bar{w}]), \\
|G(t, x, w)-G(t, x, \bar{w})| & \leq \sigma(t, V[w-\bar{w}])
\end{aligned}
$$

are satisfied on $\Sigma$.

For a function $\eta: I_{h} \rightarrow \mathbb{R}$ we write $\eta^{(r)}=\eta\left(t^{(r)}\right)$.

Now we prove a theorem on convergence of the method (9), (10). 
THEOREM 4.1. Suppose that Assumption $\mathrm{H}[\sigma, f, g, G]$ is satisfied and

1) the function $v: \Omega \rightarrow \mathbb{R}$ is a solution of (1), (2) and $v$ is of class $C^{2}$ on $\Omega$,

2) there is $c_{0}>0$ such that $h_{i} h_{j}^{-1} \leq c_{0}, i, j=1, \ldots, n$,

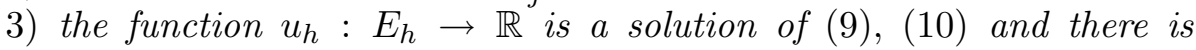
$\gamma_{0}: H \rightarrow \mathbb{R}_{+}$such that

$$
\left|v^{(r, m)}-u_{h}^{(r, m)}\right| \leq \gamma_{0}(h) \quad \text { on } \partial_{0} E_{h} \cup E_{0 . h}
$$

and $\lim _{h \rightarrow 0} \gamma_{0}(h)=0$.

Then there exists a function $\alpha: H \rightarrow \mathbb{R}_{+}$such that

$$
\left|\left(u_{h}-v_{h}\right)^{(r, m)}\right| \leq \alpha(h) \quad \text { on } E_{h} \quad \text { and } \quad \lim _{h \rightarrow 0} \alpha(h)=0
$$

where $v_{h}$ is the restriction of $v$ to the set $E_{h}$.

Proof. We divide the proof into a sequence of steps.

I. Write $z_{h}=v_{h}-u_{h}$. We construct a difference equation for $z_{h}$. Let $\Gamma_{h}: E_{h}^{\prime} \rightarrow \mathbb{R}$ and $\Gamma_{0 . h}: \partial_{0} E_{h} \cup E_{0 . h} \rightarrow \mathbb{R}$ be defined by the relations

$$
\begin{aligned}
\delta_{0} v_{h}^{(r, m)} & =F_{h}\left[v_{h}\right]^{(r, m)}+\Gamma_{h}^{(r, m)} & & \text { on } E_{h}^{\prime}, \\
v_{h}^{(r, m)} & =\varphi_{h}^{(r, m)}+\Gamma_{0 . h}^{(r, m)} & & \text { on } \partial_{0} E_{h} \cup E_{0 . h} .
\end{aligned}
$$

It follows from Lemma 1, condition 1) of the theorem and (31) that there is $\gamma: H \rightarrow \mathbb{R}_{+}$such that

$$
\left|\Gamma_{h}^{(r, m)}\right| \leq \gamma(h) \quad \text { on } E_{h}^{\prime}, \quad \lim _{h \rightarrow 0} \gamma(h)=0 .
$$

Then we have

$$
\begin{aligned}
\delta_{0} z_{h}^{(r, m)}= & \sum_{i, j=1}^{n} f_{i j}\left(P^{(r, m)}\left[u_{h}\right]\right) \delta_{i j} z_{h}^{(r+1, m)}+\sum_{i=1}^{n} g_{i}\left(P^{(r, m)}\left[u_{h}\right]\right) \delta_{i} z_{h}^{(r+1, m)} \\
& +\Lambda_{h}^{(r, m)}+\Gamma_{h}^{(r, m)}
\end{aligned}
$$

where

$$
\begin{aligned}
\Lambda_{h}^{(r, m)}= & \sum_{i, j=1}^{n}\left[f_{i j}\left(P^{(r, m)}\left[v_{h}\right]\right)-f_{i j}\left(P^{(r, m)}\left[u_{h}\right]\right)\right] \delta_{i j} v_{h}^{(r+1, m)} \\
& +\sum_{i=1}^{n}\left[g_{i}\left(P^{(r, m)}\left[v_{h}\right]\right)-g_{i}\left(P^{(r, m)}\left[u_{h}\right]\right)\right] \delta_{i} v_{h}^{(r+1, m)} \\
& +G\left(P^{(r, m)}\left[v_{h}\right]\right)-G\left(P^{(r, m)}\left[u_{h}\right]\right) .
\end{aligned}
$$


The above relations and (7), (8), (11)-(13), (23)-(25) imply

$$
\begin{aligned}
& =z_{h}^{(r, m)}+h_{0} \sum_{i=1}^{n} A_{i .+}^{(r, m)}\left[u_{h}\right] z_{h}^{\left(r+1, m+e_{i}\right)}+h_{0} \sum_{i=1}^{n} A_{i .-}^{(r, m)}\left[u_{h}\right] z_{h}^{\left(r+1, m-e_{i}\right)} \\
& +h_{0} \sum_{(i, j) \in J_{-}^{(r, m)}\left[u_{h}\right]} \frac{1}{2 h_{i} h_{j}} f_{h \cdot i j}\left(P^{(r, m)}\left[u_{h}\right]\right)\left[z_{h}^{\left(r+1, m+e_{i}-e_{j}\right)}+z_{h}^{\left(r+1, m-e_{i}+e_{j}\right)}\right] \\
& +h_{0} \sum_{(i, j) \in J_{+}^{(r, m)}\left[u_{h}\right]} \frac{1}{2 h_{i} h_{j}} f_{h \cdot i j}\left(P^{(r, m)}\left[u_{h}\right]\right)\left[z_{h}^{\left(r+1, m+e_{i}+e_{j}\right)}+z_{h}^{\left(r+1, m-e_{i}-e_{j}\right)}\right] \\
& +h_{0} \Lambda_{h}^{(r, m)}+h_{0} \Gamma_{h}^{(r, m)} .
\end{aligned}
$$

The above relation can be considered as a difference equation for the error $z_{h}$.

II. Let the function $\varepsilon_{h}^{(r)}: I_{h} \cup A_{h} \rightarrow \mathbb{R}^{+}$be defined by

$$
\varepsilon_{h}^{(r)}=\max \left\{\left|z_{h}^{(i, m)}\right|:\left(t^{(i)}, x^{(m)}\right) \in \Omega_{h} \cap\left(\left[-d_{0}, t^{(r)}\right] \times \mathbb{R}^{n}\right)\right\},-K_{0} \leq r \leq N .
$$

We will write a difference inequality for $\varepsilon_{h}$. We deduce from (16) that

$$
A_{i .+}^{(r, m)}\left[u_{h}\right] \geq 0, \quad A_{i .-}^{(r, m)}\left[u_{h}\right] \geq 0, \quad 1-A^{(r, m)}\left[u_{h}\right] \geq 0 \quad \text { for } 1 \leq i \leq n .
$$

It is easy to prove by induction with respect to $n$ that

$$
\sum_{\lambda \in \Im}\left(\frac{x-x^{(m)}}{h^{\prime}}\right)^{\lambda}\left(1-\frac{x-x^{(m)}}{h^{\prime}}\right)^{1-\lambda}=1 \quad \text { for } x^{(m)} \leq x \leq x^{(m+1)} .
$$

This gives

$$
V\left[T_{h}\left[z_{h}\right]_{[r, m]}\right](\tau) \leq T_{h_{0}}\left[\left(\varepsilon_{h}\right)_{[r]}\right](\tau), \quad \tau \in I, 0 \leq r \leq N .
$$

We conclude from condition 2) of Assumption $\mathrm{H}[\sigma, f, g, G]$ that

$$
\begin{aligned}
& \sum_{i, j=1}^{n}\left|f_{i j}\left(P^{(r, m)}\left[v_{h}\right]\right)-f_{i j}\left(P^{(r, m)}\left[u_{h}\right]\right)\right| \\
= & \sum_{i, j=1}^{n}\left|f_{i j}\left(t^{(r)}, x^{(m)},\left(T_{h}\left[v_{h}\right]\right)_{\alpha(r, m)}\right)-f_{i j}\left(t^{(r)}, x^{(m)},\left(T_{h}\left[u_{h}\right]\right)_{\alpha(r, m)}\right)\right| \\
\leq & \sigma\left(t^{(r)}, T_{h_{0}}\left[\left(\varepsilon_{h}\right)_{[r]}\right]\right)
\end{aligned}
$$

In a similar way we obtain

$$
\left\|g\left(P^{(r, m)}\left[v_{h}\right]\right)-g\left(P^{(r, m)}\left[u_{h}\right]\right)\right\| \leq \sigma\left(t^{(r)}, T_{h_{0}}\left[\left(\varepsilon_{h}\right)_{[r]}\right]\right),
$$


and

$$
\begin{aligned}
\mid G\left(t^{(r)}, x^{(m)},\left(T_{h}\left[v_{h}\right]\right)_{\alpha(r, m)}\right)-G\left(t^{(r)}, x^{(m)},\right. & \left.\left(T_{h}\left[u_{h}\right]\right)_{\alpha(r, m)}\right) \mid \\
& \leq \sigma\left(t^{(r)}, T_{h_{0}}\left[\left(\varepsilon_{h}\right)_{[r]}\right]\right) .
\end{aligned}
$$

Let $\widetilde{c} \in \mathbb{R}_{+}$be a constant such that

$$
\left|\partial_{x_{i}} v(t, x)\right|,\left|\partial_{x_{i} x_{j}} v(t, x)\right| \leq \widetilde{c}, \quad i, j=1, \ldots, n,(t, x) \in E .
$$

It follows from (26) and (33)-(37) that the function $\varepsilon_{h}$ satisfies the recurrent inequality

$$
\begin{aligned}
& \varepsilon_{h}^{(r+1)} \leq \varepsilon_{h}^{(r)}+(2 \widetilde{c}+1) h_{0} \sigma_{h}\left(t^{(r)}, T_{h_{0}}\left[\left(\varepsilon_{h}\right)_{t^{(r)}}\right]\right)+h_{0} \gamma(h), \\
& 0 \leq r \leq N-1,
\end{aligned}
$$

and

$$
\varepsilon_{h}^{(r)} \leq \gamma_{0}(h) \quad \text { for }-K_{0} \leq r \leq 0 .
$$

III. We prove that there is $\alpha: H \rightarrow \mathbb{R}_{+}$such that $\varepsilon_{h}^{(r)} \leq \alpha(h)$ for $0 \leq r \leq N$ and $\lim _{h \rightarrow 0} \alpha(h)=0$. Consider the Cauchy problem

$$
\begin{aligned}
\xi^{\prime}(t) & =(2 \widetilde{c}+1) \sigma\left(t, \xi_{t}+(\mu(h))_{t}\right)+\gamma(h), \\
\xi(t) & =\gamma_{0}(h) \quad \text { for } t \in I,
\end{aligned}
$$

where $\mu: H \rightarrow(0, \infty), \lim _{h \rightarrow 0} \mu(h)=0$ and $(\mu(h))_{t} \in C\left(I, \mathbb{R}_{+}\right)$is a constant function: $(\mu(h))_{t}(\tau)=\mu(h)$ for $\tau \in I$. It follows from condition 1) of Assumption $\mathrm{H}[f, g, \alpha]$ that there is $\widetilde{\varepsilon}>0$ such that for $\|h\| \leq \widetilde{\varepsilon}$ the maximal solution $\omega(\cdot, h)$ of $(40),(41)$ is defined on $I \cup[0, a]$ and

$$
\lim _{h \rightarrow 0} \omega(t, h)=0 \quad \text { uniformly on } I \cup[0, a] .
$$

Suppose that $\widetilde{h} \in H$ is fixed and $\|\widetilde{h}\|<\varepsilon$. Denote by $C[\widetilde{h}]$ the set of all $h \in H$ satisfying $\|h\|<\widetilde{\varepsilon}$ and $\mu(h)<\mu(\widetilde{h}), \gamma(h)<\gamma(\widetilde{h})$. Then the maximal solution $\omega(\cdot, h)$ of the difference equation (40), (41), where $h \in C[\widetilde{h}]$, satisfies the condition

$$
\omega(t, h) \leq \omega(t, \widetilde{h}) \quad \text { for } t \in I \cup[0, a] .
$$

Let $\omega_{h_{0}}(\cdot, h)$ denote the restriction of $\omega(\cdot, h): I \cup[0, a] \rightarrow \mathbb{R}_{+}$to the set $I_{h} \cup A_{h}$. It follows from (27) that

$$
T_{h_{0}}\left[\left(\omega_{h_{0}}(\cdot, h)\right)_{[r]}\right](\tau)-(\omega(\cdot, h))_{t^{(r)}}(\tau) \leq h_{0} \omega^{\prime}(a, h) \leq h_{0} \omega^{\prime}(a, \widetilde{h})
$$

for $\tau \in I$. There is $\bar{\varepsilon}>0$ such that for $h \in C[\widetilde{h}]$ with $\|h\|<\bar{\varepsilon}$ we have

$$
\mu(\widetilde{h})>\mu(h) \geq h_{0} \omega^{\prime}(a, \widetilde{h}) .
$$

We conclude from condition 1) of Assumption $\mathrm{H}[f, g, \alpha]$ and from (44), (45) 
that for $h \in C[\widetilde{h}],\|h\|<\bar{\varepsilon}$ we have

$$
\begin{aligned}
\omega^{\prime}\left(t^{(r)}, h\right)= & (2 \widetilde{c}+1) \sigma\left(t^{(r)},(\omega(\cdot, h))_{t^{(r)}}+(\mu(h))_{t}\right)+\gamma(h) \\
= & \sigma\left(t^{(r)}, T_{h_{0}}\left[\left(\omega_{h_{0}}(\cdot, h)\right)_{[r]}\right]+(\omega(\cdot, h))_{t^{(r)}}-T_{h_{0}}\left[\left(\omega_{h_{0}}(\cdot, h)\right)_{[r]}\right]\right. \\
& \left.+(\mu(h))_{t^{(r)}}\right)+\gamma(h) \\
\geq & \left.\sigma\left(t^{(r)}, T_{h_{0}}\left[\left(\omega_{h_{0}}(\cdot, h)\right)_{[r]}\right]\right)-\left(h_{0} \omega^{\prime}(a, h)\right)_{\left(t^{(r)}\right)}+(\mu(h))_{\left(t^{(r)}\right)}\right)+\gamma(h) \\
\geq & \sigma\left(t^{(r)}, T_{h_{0}}\left[\left(\omega_{h_{0}}(\cdot, h)\right)_{[r]}\right]\right)+\gamma(h), \quad 0 \leq r \leq N .
\end{aligned}
$$

Since $\omega(\cdot, h)$ is a convex function, for $h \in C[\widetilde{h}]$ with $\|h\|<\bar{\varepsilon}$ we have the difference inequality

$$
\begin{aligned}
\omega_{h_{0}}\left(t^{(r+1)}, h\right) \geq & \omega_{h_{0}}\left(t^{(r)}, h\right)+h_{0}(2 \widetilde{c}+1) \sigma\left(t^{(r)}, T_{h_{0}}\left[\left(\omega_{h_{0}}(\cdot, h)\right)_{[r]}\right]\right) \\
& +h_{0} \gamma(h), \quad 0 \leq r \leq N-1 .
\end{aligned}
$$

Since $\varepsilon_{h}$ satisfies (38), (39) the above relations and (24) imply the estimate

$$
\varepsilon_{h}^{(r)} \leq \omega\left(t^{(r)}, h\right), \quad 0 \leq r \leq N .
$$

where $h \in C[\widetilde{h}],\|h\|<\bar{\varepsilon}$. It follows from (25), (43) that the assertion of the theorem is satisfied with $\alpha(h)=\omega(a, h)$.

Remark 2. Suppose that Assumption $\mathrm{H}[\sigma, f, g]$ is satisfied with

$$
\sigma(t, w)=L\|w\|_{I}, \quad(t, p) \in[0, a] \times \mathbb{R}_{+} \text {where } L \in \mathbb{R}_{+} .
$$

Then $f, g$ and $G$ satisfy the Lipschitz condition with respect to the functional variable. We obtain the following error estimates:

$$
\left|\left(u_{h}-v_{h}\right)^{(r, m)}\right| \leq \widetilde{\alpha}(h) e^{c L a}+\widetilde{\gamma}(h) \frac{e^{c L a}-1}{c L} \quad \text { on } E_{h} \text { if } L>0,
$$

and

$$
\left|\left(u_{h}-v_{h}\right)^{(r, m)}\right| \leq \widetilde{\alpha}(h)+a \widetilde{\gamma}(h) \quad \text { on } E_{h} \text { if } L=0 .
$$

The above inequalities follow from (32) with $\alpha(h)=\omega_{h}(a)$ where $\omega_{h}$ : $[0, a] \rightarrow \mathbb{R}_{+}$is a solution of the problem

$$
\zeta^{\prime}(t)=c L \zeta(t)+\widetilde{\gamma}(h), \quad \zeta(0)=\alpha_{0}(h) .
$$

It is important in our considerations that equations with deviated variables appear in comparison problems. In the next lemma we give a suitable example.

LEMmA 2. If $\mu \geq \nu>1$ and $L \in \mathbb{R}_{+}, c \geq 1$ then the maximal solution of the Cauchy problem

$$
\zeta^{\prime}(t)=c\left[\zeta\left(t^{\mu}\right)\right]^{1 / \nu}+L \zeta(t), \quad \zeta(0)=0,
$$

is $\bar{\zeta}(t)=0$ for $t \in[0, a]$ where $a<1$. 
Proof. There are $\widetilde{\varepsilon}, \widetilde{c}>0$ such that the maximal solution $\bar{\zeta}$ of (46) satisfies

$$
\bar{\zeta}(t) \leq \widetilde{C} t \quad \text { for } t \in[0, \widetilde{\varepsilon}]
$$

Write

$$
C=\max \{c, L, \widetilde{C}\} .
$$

Then $\bar{\zeta}$ satisfies the integral inequality

$$
\zeta(t) \leq C\left[\int_{0}^{t}\left[\zeta\left(s^{\mu}\right)\right]^{1 / \nu} d s+\int_{0}^{t} \zeta(s) d s\right], \quad t \in[0, \widetilde{\varepsilon}],
$$

and $\bar{\zeta}(t) \leq C t$ for $t \in[0, \widetilde{\varepsilon}]$.

It follows from the above relations that

$$
\bar{\zeta}(t) \leq C^{k} t^{k}, \quad t \in[0, \widetilde{\varepsilon}], k \geq 1 .
$$

Then there is $\varepsilon_{0}$ such that $\bar{\zeta}=0$ for $t \in\left[0, \varepsilon_{0}\right]$ and consequently $\bar{\zeta}(t)=0$ on $[0, a]$.

REMARK 3. Note that the maximal solution of (46) with $\nu>1$ and $\mu=1$ is positive on $(0, a]$. The above considerations and examples (5), (6) show that the class of differential functional equations which is covered by our theory is more general than the corresponding classes considered in [2], [3], [13], [14].

Remark 4. Consider the explicit difference method (10)-(14). Then we need the following assumption on $f$ and on the steps of the mesh ([10]):

$$
1-2 h_{0} \sum_{j=1}^{n} \frac{1}{h_{j}^{2}} f_{j j}(P)+h_{0} \sum_{(i, j) \in J} \frac{1}{h_{i} h_{j}}\left|f_{i j}(P)\right| \geq 0,
$$

where $P \in \Sigma$. If the functions $f_{i j}, i, j=1, \ldots, n$, are bounded on $\Sigma$ then inequality (47) states relations between $h_{0}$ and $h^{\prime}=\left(h_{1}, \ldots, h_{n}\right)$. It is important in our considerations that condition (47) is omitted in the convergence theorem.

REMARK 5. Suppose that the function $\sigma$ has the structure

$$
\sigma(t, \tau)=A(t) B(\tau)
$$

where $A \in C\left([0, a], \mathbb{R}_{+}\right), B \in C\left(\mathbb{R}_{+}, \mathbb{R}_{+}\right), B(0)=0$ and $B(\tau)>0$ for $\tau>0$. The Cauchy problem

$$
\xi^{\prime}(t)=A(t) B(\xi(t)), \quad \xi(0)=0,
$$

has a unique solution if and only if

$$
\int_{0}^{\varepsilon} \frac{d \tau}{B(\tau)}=\infty, \quad \text { where } \varepsilon>0 .
$$


Then for each $c \geq 1$ the Cauchy problem

$$
\xi^{\prime}(t)=c A(t) B(\xi(t)), \quad \xi(0)=0,
$$

has the only solution $u(t)=0$ for $t \geq 0$.

There is a comparison problem

$$
\xi^{\prime}(t)=\sigma(t, \xi(t)), \quad \xi(0)=0,
$$

which has the maximal solution $u(t)=0$ for $t \geq 0$ while the initial problem

$$
\xi^{\prime}(t)=c \sigma(t, \xi(t)), \quad \xi(0)=0,
$$

for $c \geq 0$ has a positive solution on $[0, a]$. Such an example is given in [1].

\section{Numerical examples}

EXAMPLE 1. Write

$$
\begin{aligned}
E & =[0,0.25] \times[-1,1] \times[-1,1], \quad E_{0}=\{0\} \times[-1,1] \times[-1,1], \\
\partial_{0} E & =[0,0.25] \times[([-1,1] \times[-1,1]) \backslash((-1,1) \times(-1,1))] .
\end{aligned}
$$

Consider the differential equation with deviated variables

$$
\begin{aligned}
\partial_{t} z(t, x, y)= & \partial_{x x} z(t, x, y)+\partial_{y y} z(t, x, y) \\
& +x y \sin \left[z\left(t, \frac{x+y}{2}, \frac{x-y}{2}\right)-e^{-t / 2} z(t / 2, x, y)\right] \partial_{x y} z(t, x, y) \\
& +\sqrt{z\left(t^{2}, x, y\right)}-z\left(t^{2}, x, y\right)+f(t, x, y) z(t, x, y)
\end{aligned}
$$

and the initial boundary conditions

$$
\begin{array}{cl}
z(0, x, y)=1 & \text { for }(x, y) \in[-1,1] \times[-1,1], \\
z(t,-1, y)=z(t, 1, y)=e^{t y^{2}} & \text { for } t \in[0,0.25], y \in[-1,1], \\
z(t, x,-1)=z(t, x, 1)=e^{t x^{2}} & \text { for } t \in[0,0.25], x \in[-1,1] .
\end{array}
$$

where

$$
f(t, x, y)=-4 t-4 t^{2}\left(x^{2}+y^{2}+x^{2} y^{2}\right)+\left(x^{2}+y^{2}-1\right) .
$$

The solution of (48)-(51) is known:

$$
v(t, x, y)=e^{t\left(x^{2}+y^{2}-1\right)} .
$$

We found approximate solutions of (48), (49) using both implicit and explicit numerical methods, and taking the following steps of the mesh: $h_{0}=0.0025, h_{1}=0.0025, h_{2}=0.0025$.

Let $u_{h}$ denote the approximate solution of (48)-(51) which is obtained by a difference scheme. 

mula:

The average errors of the method $\varepsilon_{h}^{(r)}$ are found with the following for-

$$
\varepsilon_{h}^{(r)}=\frac{1}{\left(2 N_{1}+1\right)\left(2 N_{2}+1\right)} \sum_{i=-N_{1}}^{N_{1}} \sum_{j=-N_{2}}^{N_{2}}\left|u_{h}^{(r, i, j)}-v_{h}^{(r, i, j)}\right|
$$

where $N_{1} h_{1}=1, N_{2} h_{2}=1$ and $v_{h}$ is the restriction of the function $v$ to the mesh.

Note that the function $f$ and the steps of the mesh do not satisfy condition (47), which is necessary for the explicit method to be convergent. In our numerical example the average errors of the explicit method exceeded $10^{17}$, while the average errors $\varepsilon_{h}$ for fixed $t^{(r)}$ of implicit method are given in Table 1.

Table 1. Errors $\left(\varepsilon_{h}\right)$

$$
\left(h_{0}=0.0025, h_{1}=0.0025, h_{2}=0.0025\right)
$$

\begin{tabular}{cc}
\hline$t^{(r)}$ & $\varepsilon_{h}^{(r)}$ \\
\hline$t=0.05$ & $33 \cdot 10^{-5}$ \\
$t=0.01$ & $55 \cdot 10^{-5}$ \\
$t=0.15$ & $70 \cdot 10^{-5}$ \\
$t=0.20$ & $80 \cdot 10^{-5}$ \\
$t=0.25$ & $86 \cdot 10^{-5}$ \\
\hline
\end{tabular}

The differential equation (48) contains the deviated variables $(t,(x+y) / 2$, $(x-y) / 2)$ and the example has the following property: if $\left(t^{(r)}, x^{\left(m_{1}\right)}, y^{\left(m_{2}\right)}\right)$ is a grid point then

$$
\left(t^{(r)}, 0.5\left(x^{\left(m_{1}\right)}+y^{\left(m_{2}\right)}\right), 0.5\left(x^{\left(m_{1}\right)}-y^{\left(m_{2}\right)}\right)\right),
$$

in general, is not a grid point. We approximate the value $z\left(t^{(r)}, 0.5\left(x^{\left(m_{1}\right)}+\right.\right.$ $\left.\left.y^{\left(m_{2}\right)}\right), 0.5\left(x^{\left(m_{1}\right)}-y^{\left(m_{2}\right)}\right)\right)$ using the interpolating operator $T_{h}$ with $n=2$.

Example 2. Consider the integral-differential equation

$$
\begin{aligned}
& \partial_{t} z(t, x, y) \\
& =\left\{1+\left[2 t\left(y^{2}-1\right) \int_{-1}^{x} s z(t, s, y) d s-z(t, x, y)\right]^{2}\right\} \partial_{x x} z(t, x, y) \\
& \quad+\left\{1+\left[2 t\left(x^{2}-1\right) \int_{-1}^{y} s z(t, x, s) d s-z(t, x, y)\right]^{2}\right\} \partial_{y y} z(t, x, y) \\
& \quad+\partial_{x y} z(t, x, y) \sin \left[\left(x^{2}-1\right)\left(y^{2}-1\right) \int_{0}^{t} z(\tau, x, y) d \tau-z(t, x, y)+1\right] \\
& \quad+f(t, x, y) z(t, x, y)
\end{aligned}
$$


and the initial boundary condition

$$
z(t, x, y)=1 \quad \text { on } \partial_{0} E \cup E_{0}
$$

where $E, E_{0}, \partial E_{0}$ are defined as earlier and

$$
f(t, x, y)=-4 t\left(x^{2}+y^{2}-2\right)-8 t^{2}\left(x^{2}\left(y^{2}-1\right)^{2}+y^{2}\left(x^{2}-1\right)^{2}\right) .
$$

The solution of (52), (53) is known:

$$
v(t, x, y)=e^{t\left(x^{2}-1\right)\left(y^{2}-1\right)} .
$$

As in the previous numerical example we chose the steps of the mesh which do not satisfy condition (47). In accordance with our expectations the explicit method is not convergent, and the average errors are so big that it is impossible for the personal computer to compute them, while the implicit method is convergent and gives the following average errors.

Table 2. Errors $\left(\varepsilon_{h}\right)$

$$
\left(h_{0}=0.0025, h_{1}=0.0025, h_{2}=0.0025\right)
$$

\begin{tabular}{cc}
\hline$t^{(r)}$ & $\varepsilon_{h}^{(r)}$ \\
\hline$t=0.10$ & $15 \cdot 10^{-4}$ \\
$t=0.20$ & $69 \cdot 10^{-4}$ \\
$t=0.30$ & $18 \cdot 10^{-3}$ \\
$t=0.40$ & $38 \cdot 10^{-3}$ \\
$t=0.50$ & $66 \cdot 10^{-3}$ \\
\hline
\end{tabular}

The differential equation (52) contains integrals of the unknown function $z$. Therefore the corresponding difference equation includes the terms

$$
\int_{-x^{\left(m_{1}\right)}}^{x^{\left(m_{1}\right)}} \tau z\left(t^{(r)}, \tau, y\right) d \tau, \quad \int_{-y^{\left(m_{2}\right)}}^{y^{\left(m_{2}\right)}} \tau z\left(t^{(r)}, x, \tau\right) d \tau, \quad \int_{0}^{t^{(r)}} z\left(\tau, x^{\left(m_{1}\right)}, y^{\left(m_{2}\right)}\right) d \tau
$$

where $z\left(t^{(r)}, x^{\left(m_{1}\right)}, y^{\left(m_{2}\right)}\right)$ is a grid point. The above integrals are approximated by

$$
\begin{gathered}
\int_{-x^{\left(m_{1}\right)}}^{x^{\left(m_{1}\right)}} \tau T_{h}\left[z_{h}\right]\left(t^{(r)}, \tau, y\right) d \tau, \quad \int_{-y^{\left(m_{2}\right)}}^{y^{\left(m_{2}\right)}} \tau T_{h}\left[z_{h}\right]\left(t^{(r)}, x, \tau\right) d \tau, \\
\int_{0}^{t^{(r)}} T_{h}\left[z_{h}\right]\left(\tau, x^{\left(m_{1}\right)}, y^{\left(m_{2}\right)}\right) d \tau
\end{gathered}
$$

where $z_{h}$ is a solution of a difference equation. The above method is equivalent to the trapezoidal rule.

The computation was performed on a PC computer. Numerical results are consistent with our mathematical theory. 
Difference schemes obtained by a discretization of problem (1), (2) have the following property: a large number of previous values $z^{(r, m)}$ must be preserved, because they are needed to compute an approximate solution with $t=t^{(r+1)}$.

The above examples show that there are implicit difference schemes which are convergent, while the corresponding classical methods are not convergent. This is due to the fact that we need the relation (47) for steps of the mesh in the classical case. We do not need this condition in our implicit method. Implicit difference methods in Sections 5 and 6 have the potential for applications in the numerical solving of differential integral equations or equations with deviated variables.

\section{References}

[1] A. Augustynowicz, Some remarks on comparison functions, submitted.

[2] R. Ciarski, Stability of difference equations generated by parabolic differential functional equations, Demonstratio Math. 38 (2005), 101-117.

[3] W. Czernous and Z. Kamont, Implicit difference methods for parabolic functional differential equations, Z. Angew. Math. Mech. 85 (2005), 326-338.

[4] K. Gopalsamy and P. X. Weng, On the dynamics of a periodic delay logistic equation with diffusion, Bull. Austral. Math. Soc. 45 (1992), 113-134.

[5] -, - Global attractivity and "level crossings" in a periodic partial integrodifferential equation, Houston J. Math. 18 (1992), 551-575.

[6] G. Hetzer, A functional reaction-diffusion equation from climate modelling: S-shapedness of the principal branch of fixed points of the time-1-map, Differential Integral Equations 8 (1995), 1047-1059.

[7] Z. Kamont, Hyperbolic Functional Differential Inequalities and Applications, Kluwer, Dordrecht, 1999.

[8] Z. Kamont and H. Leszczyński, Stability of difference equations generated by parabolic differential-functional problems, Rend. Mat. Appl. (7) 16 (1996), 265-287.

[9] J. M. Mahaffy and C. V. Pao, Models of genetic control by repression with time delays and spatial effects, J. Math. Anal. Appl. 20 (1984), 39-57.

[10] M. Malec, Sur une famille bi-paramétrique des schémas des différences finies pour un système d'équations paraboliques aux dérivées mixtes et avec des conditions aux limites du type de Neumann, Ann. Polon. Math. 32 (1976), 33-42.

[11] M. Malec and M. Rosati, Weak monotonicity for nonlinear systems of functionalfinite difference inequalities of parabolic type, Rend Mat. (7) 3 (1983), 157-170.

[12] M. Malec et A. Schiaffino, Méthode aux différences finies pour une équation nonlinéaire différentielle fonctionnelle du type parabolique avec une condition initiale de Cauchy, Boll. Un. Mat. Ital. B (7) 1 (1987), 99-109.

[13] C. V. Pao, Coupled nonlinear parabolic systems with time delays, J. Math. Anal. Appl. 196, (1995), 237-265.

[14] - Numerical methods for systems of nonlinear parabolic equations with time delays, ibid. 240 (1999), 249-279.

[15] —, Finite difference reaction diffusion systems with coupled boundary conditions and time delay, ibid. 272 (2002), 407-434.

[16] W. Walter, Differential and Integral Inequalities, Springer, Berlin, New York, 1970. 
[17] P. K. C. Wang and M. L. Bandy, Stability of distributed-parameter systems with time-delays, J. Electronics Control 15 (1963), 342-362.

[18] J. Wu, Theory and Applications of Partial Functional Differential Equations, Springer, New York, 1996.

Institute of Mathematics

University of Gdańsk

Wit Stwosz St. 57

80-952 Gdańsk, Poland

E-mail: karolina.kropielnicka@math.univ.gda.pl

Received on 15.5.2007;

revised version on 17.9.2007 
